\title{
Therapy concepts in the context of precision medicine for pediatric malignancies - children are not adults
}

\author{
Elisabeth Salzer (D) C Caroline Hutter
}

Received: 17 May 2021 / Accepted: 26 July 2021 / Published online: 3 September 2021

(C) The Author(s) 2021

Summary Cancer remains the leading cause of death from disease among children beyond the age of one. Survival of pediatric patients with cancer has dramatically improved over the last decades but some tumors remain almost intractable and relapse is still associated with an infaust prognosis. Despite the heterogeneity of pediatric malignancies, most treatments include the same set of generic therapies. Optimizing delivery of conventional therapeutics has been the driving force behind continuous improvements but further escalation of conventional therapy is unlikely to improve outcomes. The limited success of targeted drugs in pediatric cancer patients, originally developed for cancers in adults, can be connected to the different etiology of tumors in children versus adults. In addition, many pediatric cancers lack reliable biomarkers, cannot be studied in large cohorts and only few available therapies target abberations specific for certain pediatric cancers.

These observations have led to the establishment of pediatric precision-medicine programs. The major goal of these programs is to identify patienttailored molecular treatment plans that will eventually improve quality of life and survival. Despite the initial euphemism, the impact of actionable matched treatments and the most adequate value-based genomics strategies are not yet well established. A noncompetitive collaborative model based on pediatric cancer priorities and strong collaboration between academia, pharmaceutical companies and regulators is needed. In the near future, clinical trials need

\section{E. Salzer · C. Hutter $(\bowtie)$}

St. Anna Children's Hospital, Department of Pediatrics, Medical University of Vienna, Vienna, Austria caroline.hutter@stanna.at

\section{E. Salzer}

elisabeth.salzer@stanna.at to focus on biologically defined patient subsets, in an even smaller patient population. A major collaborative effort between all associated groups will be necessary to ensure success of pediatric precision cancer medicine.

Keywords Pediatric oncology · High throughput genomic profiling - Collaborative innovative trial design

Despite major progress over the last 50 years cancer remains the leading cause of death from disease among children beyond the age of one with approximately 2.5 newly diagnosed patients per 100,000 individuals [1]. Due to both cooperative protocols and multidisciplinary treatment, overall survival of pediatric cancer patients increased dramatically. Up to the early 1970 s, the 10 -year survival rate of patients younger than 20 years was below $20 \%$. Today, $83 \%$ of these patients are alive 10 years after initial diagnosis. This overall success story of pediatric oncology is largely due to excellent outcomes in the treatment of more common cancers [2]. The rate of progress has been greater in hematologic malignances, neuroblastoma, gonadal cancers, and some central nervous system tumors than in other solid tumors, where outcomes have almost plateaued over the last decade [3]. Still, several childhood solid tumors remain intractable-especially metastatic disease is hard to treat and is associated with a poor outcome [4]. In addition, $20 \%$ of pediatric cancer patients relapse (corresponding to 400 patients/year in Germany or $40 /$ year in Austria) and of those only about $10 \%$ can be cured $[2,5]$.

Despite the complexity and variability of pediatric cancers, most are treated with the same set of generic therapies: surgery, radiation therapy, chemotherapy, 
and immunotherapy [6]. Optimizing delivery of conventional therapeutics has been the driving force behind continuous improvements in pediatric cancer survival over the last 40 years. However, further escalation of conventional therapy is unlikely to yield improvement in cancers that currently have unacceptably low cure rates or patients with relapsed or primary metastatic disease [6]. In the current era where an unprecedented number of targeted and immuno-oncology drugs have been developed for adult cancers [7, 8], high hopes have been placed also in pediatric oncology as a mean to bridge that gap. However, comparably few successful examples have been directly implemented into frontline childhood cancer care: rituximab for Burkitt lymphoma, blinatumomab for acute lymphoblastic leukemia, imatinib for chronic myeloid leukemia, sorafenib for FLT3-mutated acute myeloid leukemia, dinutuximab and anaplastic lymphoma kinase (ALK) inhibitors for high-risk neuroblastoma and others. The limited success can be connected to the absence of reliable biomarkers, the lack of large cohorts, limited longitudinal studies, and the few available targeted therapies specific for children with cancer.

As opposed to the predominance of epithelial origin in adult tumors, pediatric cancers are diverse in their origin and are now considered as diseases of dysregulated development [9]. Revolutionary advances in next-generation sequencing technology [10-12] together with progress in chromatin and stem cell biology [13] have piloted in a new molecular understanding of childhood cancer. Evidence from mouse models of childhood cancer suggest malignant transformation in stem or progenitor cells during developmental time windows, rather than numerous mutational "hits" as frequently observed in adult cancers [14-19]. Fusion genes are more common in pediatric than in adult cancers, and certain mutations found are almost exclusive to pediatric tumors indicative of potentially novel oncogenic pathways [11, 20, 21].

These observations have prompted regulatory initiatives in Europe (The European Pediatric Medicine Regulation, 2006) and the US (Best Pharmaceutical Act, 2002; Pediatric Research Equity Act, 2003) to promote pediatric precision-medicine programs. Personalized cancer therapy is not a new concept, particularly in pediatric oncology. Established pediatric protocols already stratify patients into different risk groups according to therapy response, age and gender, disease localization, biomarkers and many other factors [22]. However, in contrast to conventional chemotherapy, which destroys both tumor and normal cells, precision medicine aims at uncovering and specifically targeting the molecular alteration of the malignancy [23]. Over the last few years several national and international programs such as MAPPYACTS [24], INFORM [25], ZERO [26], PROFYLE [27] and the TARGET [28] initiative have evolved. The unifying concept of these programs is to biologically characterize tumor samples especially for patients with relapsed or refractory high-risk disease for which no standard of care therapy is available. State-of-theart next-generation sequencing technologies are used to define a "fingerprint" of each individual tumor. In an interdisciplinary panel experienced pediatric oncologists, bioinformaticians, biologists, and pharmacologists classify and weigh the identified aberrations/targets found for each single patient according to clinical relevance and available targeted therapies (Figure 1). The major goal of these programs is to identify patient-tailored molecular treatment plans that will eventually improve quality of life and survival.

However, despite the initial euphemism, the feasibility of large-scale clinical use of precision medicine, the impact of actionable matched treatments and the most adequate value-based genomics strategies are not yet well established [29]. It is key to inform parents and patients upfront about relatively low rates of sustained clinical responses and potential limitations of targeted therapies due to still not yet fully recognized and predictable side effects. Furthermore, difficulties in interpreting sequencing results and their consequences for bedside medicine remain an important issue. Often, in depth tumor characterization reveals mechanistic differences in conventional entities, generates more heterogeneity and makes rare diseases even rarer. Due to their inherent pre- and post-analytical complexity interpretation and validation of precision oncology studies is especially challenging, as epidemiological and genetic variability between populations, individual tumor heterogeneities, sequencing techniques, genomic panels covered, and bioinformatics analysis differ widely between the trials and commercially available platforms [30]. First reports of three independent pediatric precision cancer medicine programs showed limited potential of genomics alone to guide selection of targeted therapies: The PEDS-MIONCOSEQ [31] and INFORM [32] studies demonstrate that a small set of patients $(n=9$ [10\%] and 2 [4\%], respectively) had a clinical response following initiation of a targeted therapy. In the iCat [33] study, which included over 100 patients, the 3 patients who received a targeted therapy failed to show objective responses. However, later, larger studies could identify subgroups benefitting from molecularly matched targeted treatment (Tilburg, abstract ASCO 2021 [25]). To increase the predictive value, most of these programs now include drug sensitivity and resistance profiling (DSRP) on either patient cells or patient-derived xenografts. In combination with genomic profiling, DSRP can generate a more comprehensive understanding of the tumor, allowing appropriate therapy selection for each individual patient [34].

Consequently, pediatric cancer is to be treated in a more individualized way and clinical trials need to focus on biologically defined patient subsets, in an 


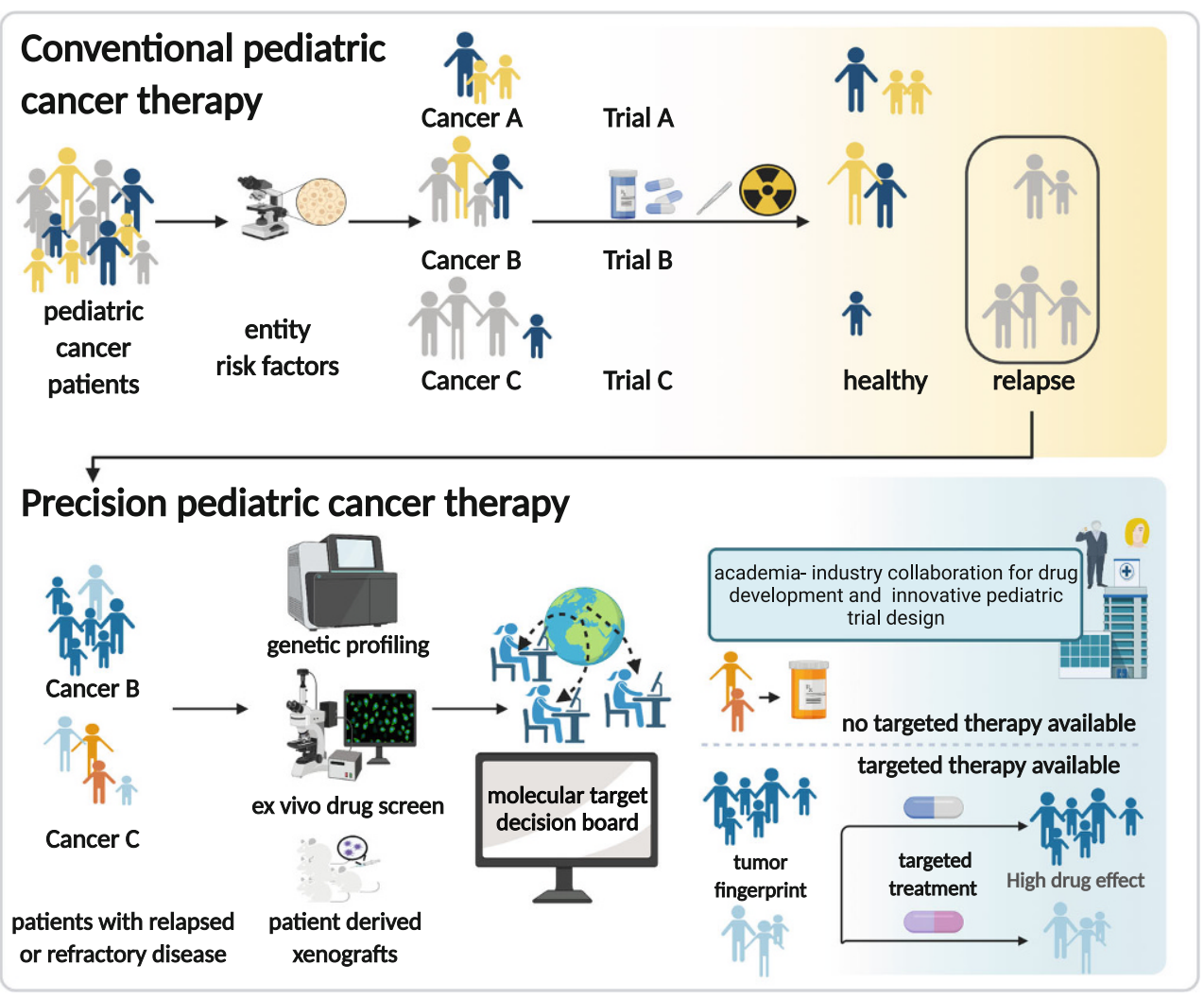

Fig. 1 Graphical display of conventional versus precision cancer therapy for refractory and relapsed pediatric patients. Conventional chemotherapy (top) relies on entity specific treatments derived from a combination of histopathology and biomarkers. Patients are treated according to standardized international protocols, which mainly include a combination of chemotherapy, radiation, surgery, and immune/targeted therapy for selected cases. Precision cancer therapy (bottom) uses genomic profiling (genome, exome, RNA, and methylation sequencing) of tissue from each individual cancer patient in combination with experimental data on patient derived tumor cells using xenograft models or ex vivo drug testing. Gen-

even smaller patient population. National and international collaborative studies are therefore required to attain sufficient patients and complete trials in a timely manner. The key challenge is to design trials that can clearly delineate the effect of the new agent under study. Innovative adaptive study design is needed, based on the underlying cancer biology rather than certain cancer types [35]. Furthermore, new endpoints that utilize functional imaging or molecular biomarkers should be incorporated into clinical trials as a measure of response and MRD. To achieve this ambitious goal, cooperation with regulatory bodies, pharmaceutical enterprises, parents, and patients is key. However, childhood cancer is considered a rare disease and the market for pediatric cancer treatments is small. In addition, several noncommercial barriers have also been highlighted as possible blocks to drug development in childhood cancer: rare populations leading to slow accrual and small samples sizes for conventional trial designs, erated data is discussed in a multidisciplinary molecular tumor board, a targeted treatment plan including potential biomarkers for response evaluation are selected for every patient. The patient is enrolled in a clinical study. For patient groups where no targeted therapy is available a collaboration with industry for drug development or pediatric clinical trial design is initiated. At present, precision medicine options are mainly available for refractory and relapsed $(R \& R)$ patients but the goal is to offer precision medicine approaches to all patients also during first-line therapy. This figure was created with BioRender.com and is not included under the Creative Commons CC BY license of this publication

the need for suitable pediatric formulations (tablets and capsules are less appropriate than intravenous or liquid formulations), concerns for both acute and long-term safety in the developing child and anxiety over real and perceived ethical difficulties in studying new agents in this vulnerable population [36]. In addition, there is a smaller number of druggable genetic aberrations in childhood cancers and pharmacokinetics/dynamics differ significantly between infants, adolescents, and young adults. In this system drugs that are potentially of great interest in pediatrics, but without an adult indication are not likely to be tested in pediatric clinical trials. This is of particular concern since the pathogenesis of pediatric cancer is largely different from adult cancer, as stated above.

To overcome this issue a non-competitive collaborative model based on pediatric cancer priorities and strong collaboration between academia, pharmaceutical companies and regulators is needed. In this scenario the pediatric oncology academic community 
performs preclinical testing and selects the strongest candidates for clinical trials together with pharmaceutical companies and regulators. This would allow promising novel therapies to be studied in parallel and sequentially using innovative biomarker-led trial designs, (e.g., multi-arm Bayesian adaptive designs) to allow for more feasible, efficient, and expedited clinical trials [37]. The Innovative Therapies for Children with Cancer (ITCC) Consortium [38] is an example of such an institution. The ITCC is a non-profit organization and gathers 63 European pediatric oncology departments with expertise in conducting early phase trials in children and adolescents, and 25 European research laboratories. The aim of this organization is to develop novel therapies for the treatment of pediatric and adolescent cancers in cooperation with regulatory bodies, pharmaceutical enterprises, parents, and patients. Since its foundation in 2003 the ITCC has paved the way for advancing pediatric cancer therapy.

As we continue into the 21st century, our increased understanding of the molecular and genetic basis of childhood cancer will facilitate further refinement of risk-adapted therapy that utilizes molecular and genetic signatures for risk stratification. A major collaborative effort between all associated groups-clinicians, scientists, insurers, biopharmaceutical companies, regulators, and patients-will be necessary to keep driving pediatric precision cancer medicine forward and make it a viable field that benefits all [39].

\section{Take home message}

- Childhood cancers are biologically different from adult cancer entities and need different therapy concepts.

- A major collaborative effort between all stakeholders is needed.

Funding Open access funding provided by Medical University of Vienna.

Conflict of interest E. Salzer and C. Hutter declare that they have no competing interests.

Open Access This article is licensed under a Creative Commons Attribution 4.0 International License, which permits use, sharing, adaptation, distribution and reproduction in any medium or format, as long as you give appropriate credit to the original author(s) and the source, provide a link to the Creative Commons licence, and indicate if changes were made. The images or other third party material in this article are included in the article's Creative Commons licence, unless indicated otherwise in a credit line to the material. If material is not included in the article's Creative Commons licence and your intended use is not permitted by statutory regulation or exceeds the permitted use, you will need to obtain permission directly from the copyright holder. To view a copy of this licence, visit http://creativecommons.org/licenses/by/4.0/.

\section{References}

1. Norris RE, Adamson PC. Challenges and opportunities in childhood cancer drug development. Nat Rev Cancer. 2012;12:776-82.

2. Universitätsmedizin der Johannes Gutenberg-Universität Mainz. Deutsches Kinderkrebsregister. 2021. http://www. kinderkrebsregister.de. Accessed: 15. May2021.

3. Pritchard-Jones K, Hargrave D. Declining childhood and adolescent cancer mortality: great progress but still much to be done. Cancer. 2014;120:2388-91.

4. Smith MA, et al. Outcomes for children and adolescents with cancer: challenges for the twenty-first century. J Clin Oncol. 2010;28:2625-34.

5. StatistikAustria. Krebs bei Kindernund Jugendlichen. 2021. https://www.statistik.at/web_de/statistiken/menschen_ und_gesellschaft/gesundheit/krebserkrankungen/krebs_ bei_kindern-und_jugendlichen/index.html. Accessed: 15 . May 2021.

6. Saletta F, Seng MS, Lau LMS. Advances in paediatric cancer treatment. Transl Pediatr. 2014;3:156-82.

7. Murciano-Goroff YR, Warner AB, Wolchok JD. The future of cancer immunotherapy: microenvironment-targeting combinations. Cell Res. 2020;30:507-19.

8. TrendsTalk. The future of cancer research. Trends Cancer. 2020;6:724-9.

9. RahalZ,AbdulhaiF,KadaraH,SaabR.Genomics ofadultand pediatric solid tumors. Am J Cancer Res. 2018;8:1356-86.

10. Dharia NV, et al. A first-generation pediatric cancer dependency map. Nat Genet. 2021;53:529-38.

11. Gröbner SN, Worst BC, WeischenfeldtJ, etal. Thelandscape of genomic alterations across childhood cancers. Nature. 2018;555:321-7.

12. MaX, etal. Pan-cancergenome and transcriptome analyses of 1,699 paediatric leukaemias and solid tumours. Nature. 2018;555:371-6.

13. Filbin M, Monje M. Developmental origins and emerging therapeutic opportunities for childhood cancer. Nat Med. 2019;25:367-76.

14. Li Z, et al. Developmental stage-selective effect of somatically mutated leukemogenic transcription factor GATA1. NatGenet. 2005;37:613-9.

15. Huntly BJP, et al. MOZ-TIF2, but not BCR-ABL, confers properties of leukemic stem cells to committed murine hematopoietic progenitors. Cancer Cells. 2004;6:587-96.

16. Funato K, Major T, Lewis PW, Allis CD, Tabar V. Use of human embryonic stem cells to model pediatric gliomas with H3.3K27M histone mutation. Science. 2014;346:1529-33.

17. Han Z-Y, et al. The occurrence of intracranial rhabdoid tumours in mice depends on temporal control of Smarcb1 inactivation. NatCommun. 2016;7:10421.

18. Ng JMY, et al. Generation of a mouse model of atypical teratoid/rhabdoid tumor of the central nervous system through combined deletion of Snf5 and p53. Cancer Res. 2015;75:4629-39.

19. Vitte J, Gao F, Coppola G, Judkins AR, Giovannini M. Timing of Smarcb1 and Nf2 inactivation determines schwannoma versus rhabdoid tumor development. Nat Commun. 2017;8:300.

20. Vogelstein B, et al. Cancer genome landscapes. Science. 2013;339:1546-58.

21. Schwartzentruber J, et al. Driver mutations in histone H3.3 and chromatin remodelling genes in paediatric glioblastoma. Nature. 2012;482:226-31.

22. Burdach SEG, Westhoff M-A, Steinhauser MF, Debatin K-M. Precision medicine in pediatric oncology. Mol Cell Pediatr. 2018;5:6. 
23. Collins FS, Varmus H. A new initiative on precision medicine. NEngl J Med. 2015;372:793-5.

24. Geoerger B, et al. Abstract CT004: European pediatric precision medicine program in recurrent tumors: first results from MAPPYACTS molecular profiling trial towards AcSe-ESMART proof-of-concept study. Cancer Res. 2017;77(13 Supplement):CT4. https://doi.org/10.1158/ 1538-7445.AM2017-CT004.

25. van Tilburg CM, et al. The pediatric precision oncology study INFORM: Clinical outcome and benefit for molecular subgroups. JClin Oncol. 2020;38:LBA10503.

26. Hadjadj D, Deshmukh S, Jabado N. Entering the era of precision medicine in pediatric oncology. Nat Med. 2020;26:1684-5.

27. Terry Fox Research Institute. Precision oncology for young people (Profyle). 2019. https://www.tfri.ca/ our-research/research-project/precision-oncology-foryoung-people-(profyle). Accessed: 15. May 2021.

28. National Cancer Institute. TARGET: therapeutically applicable research to generate effective treatments. 2021. http://ocg.cancer.gov/programs/target. Accessed: 15. May 2021

29. Tannock IF, Hickman JA. Limits to personalized cancer medicine. NEngl J Med. 2016;375:1289-94.

30. Vasconcellos VF, Colli LM, Awada A, de Castro GJr. Precision oncology: as much expectations as limitations. Ecancermedicalscience. 2018;12:ed86.

31. Mody RJ, et al. Integrative clinical sequencing in the management of refractory or relapsed cancer in youth. JAMA. 2015;314:913.

32. Worst BC, et al. Next-generation personalised medicine for high-risk paediatric cancer patients-the INFORM pilot study. Eur J Cancer. 2016;65:91-101.
33. Harris MH, et al. Multicenter feasibility study of tumor molecular profiling to inform therapeutic decisions in advanced pediatric solid tumors: the Individualized Cancer Therapy (iCat) study. JAMA Oncol. 2016;2:608.

34. Tsoli M, et al. Integration of genomics, high throughput drug screening, and personalized xenograft models as a novel precision medicine paradigm for high risk pediatric cancer. Cancer BiolTher. 2018;19:1078-87.

35. Li A, Bergan RC. Clinical trial design: past, present, and future in the context of big data and precision medicine. Cancer. 2020;126:4838-46.

36. Boklan J. Little patients, losing patience: pediatric cancer drug development. Mol Cancer Ther. 2006;5:1905-8.

37. AngeliniP,Pritchard-JonesK, HargraveDR.Challengesinincentivizing the pharmaceutical industry to supporting pediatric oncology clinical trials. Clin Investig. 2013;3:101-3.

38. ITCC. Web site. 2021. https://www.itcc-consortium.org. Accessed: 15. May 2021.

39. Chow EJ, et al. New agents, emerging late effects, and the development of precision survivorship. J Clin Oncol. 2018;36:2231-40.

Publisher's Note Springer Nature remains neutral with regard to jurisdictional claims in published maps and institutional affiliations.

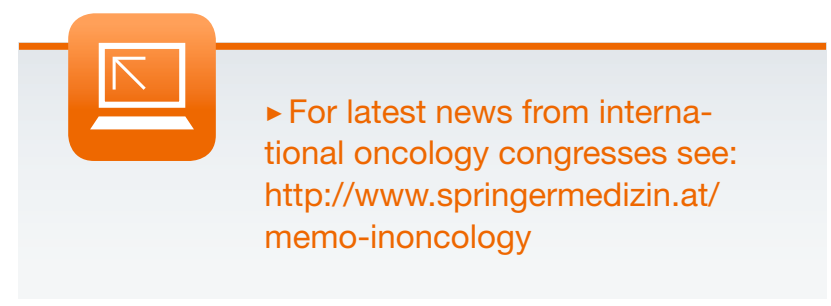

